\title{
Studying the dynamics of the gut microbiota using metabolically stable isotopic labeling and metaproteomics.
}

\section{CURRENT STATUS: POSTED}

Research Square

Patrick Smyth

University of Ottawa

ORCiD: https://orcid.org/0000-0003-3135-3354

Xu Zhang

University of Ottawa

Zhibin Ning

University of Ottawa

Janice Mayne

University of Ottawa

Jasmine I. Moore

University of Ottawa

Krystal Walker

University of Ottawa

Mathieu Lavallée-Adam

University of Ottawa

Daniel Figeys

dfigeys@uottawa.caCorresponding Author

ORCiD: https://orcid.org/0000-0002-5373-7546

DOI:

10.21203/rs.3.rs-17324/v1

\section{SUBJECT AREAS}

General Microbiology

\section{KEYWORDS}

microbiome, metaproteomics, dynamics, stable isotope, partial labeling, 15N labeling 
Abstract

Background: The gut microbiome and its metabolic processes are dynamic systems. Surprisingly, our understanding of gut microbiome dynamics is limited. Here we report a metaproteomic workflow that involves protein stable isotope probing (protein-SIP) and identification and quantification of partially labeled peptides. We also developed a package, which we call MetaProfiler, that corrects for false identifications and performs phylogenetic and time series analysis for the study of gut microbiome functional dynamics.

Results: From the stool sample of five mice that were fed with lensuremath $\{\wedge\{15\}\} N$ hydrolysate from Itextit\{Ralstonia eutropha\}, we identified 15,297 non-redundant unlabeled peptides of which 10,839 of their heavy counterparts were quantified. These peptides revealed incorporation profiles over time that were different between and within taxa, as well as between and within clusters of orthologous groups (COGs).

Conclusions: Our study helps unravel the complex dynamics of protein synthesis and bacterial dynamics in the mouse microbiome. Availability: MetaProfiler and the bioinformatic pipeline are available at https://github.com/psmyth94/MetaProfiler.git.

\section{Full-text}

Due to technical limitations, full-text HTML conversion of this manuscript could not be completed. However, the manuscript can be downloaded and accessed as a PDF.

Figures 


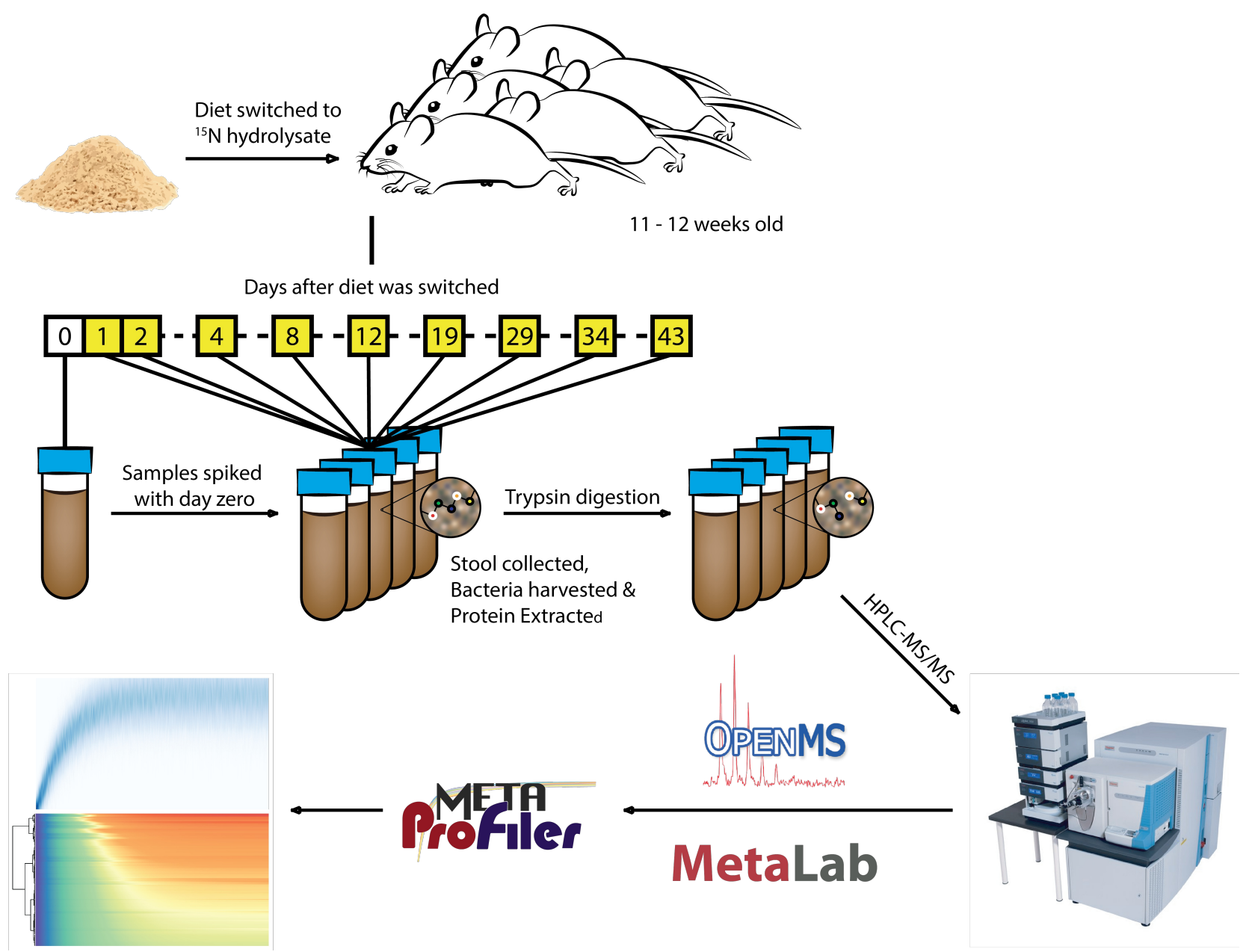

Figure 1

Figure 1 Overview of Experimental Workflow Five 11-12 weeks old male mice were fed with a $15 \mathrm{~N}$ labeled mouse diet, where hydrolysate of the chemolithoautotrophic bacteria, Ralstonia eutropha, was the source of heavy nitrogen. Stool samples were collected at 10 dierent time points, were spiked with day 0 microbiome, and were processed by mass spectrometry. The mass spectrometric data was analyzed by OpenMS, MetaLab, and MetaProler to identify and quantify peptides and proteins and to establish proles of $15 \mathrm{~N}$ incorporations over time. 
A

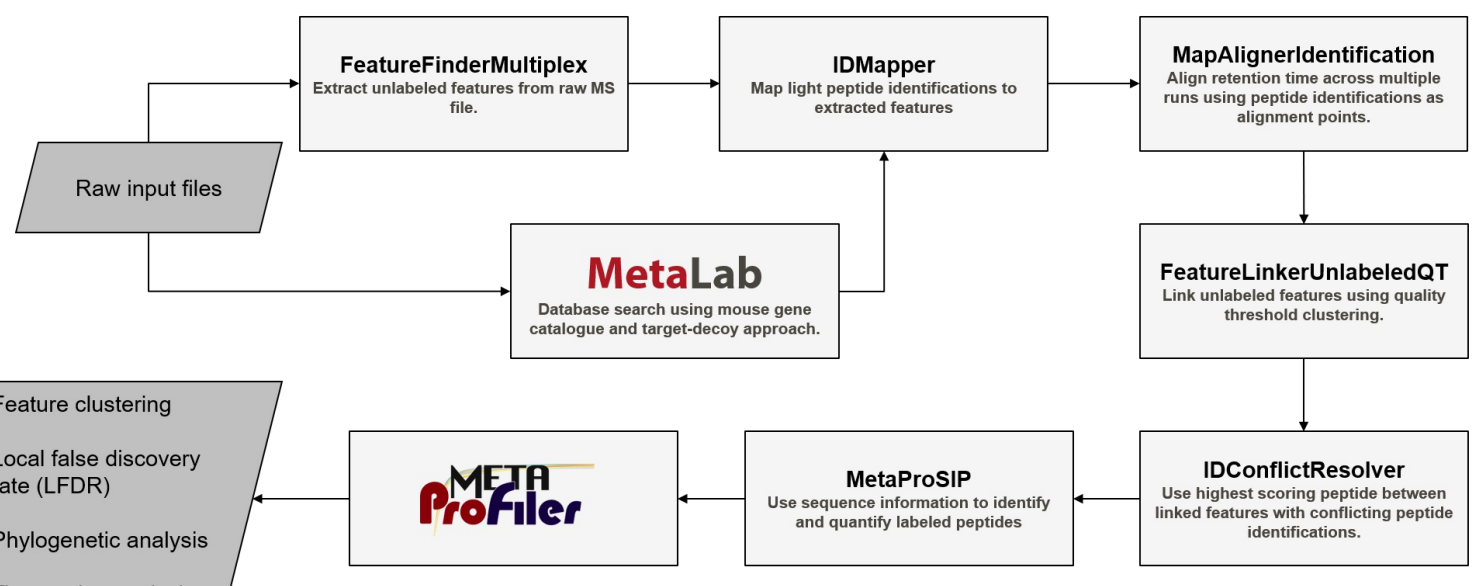

- Time series analysis

B

AADDAAGLTISEK in Sample 4 on Day 4
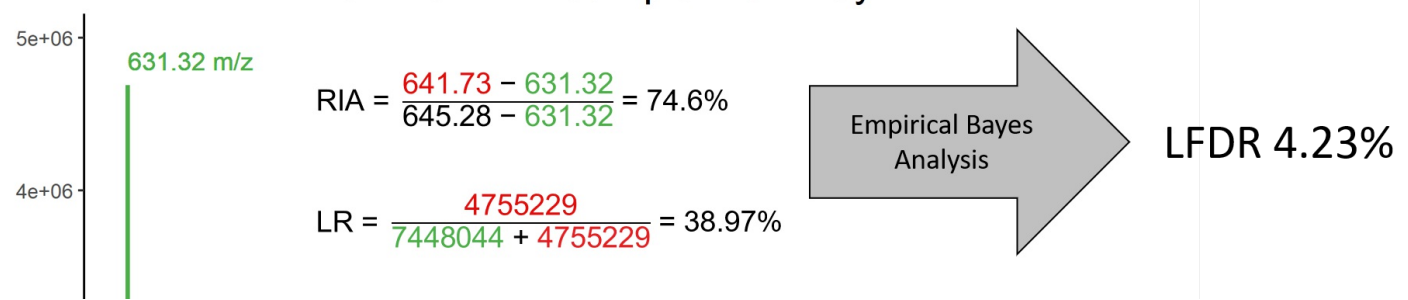


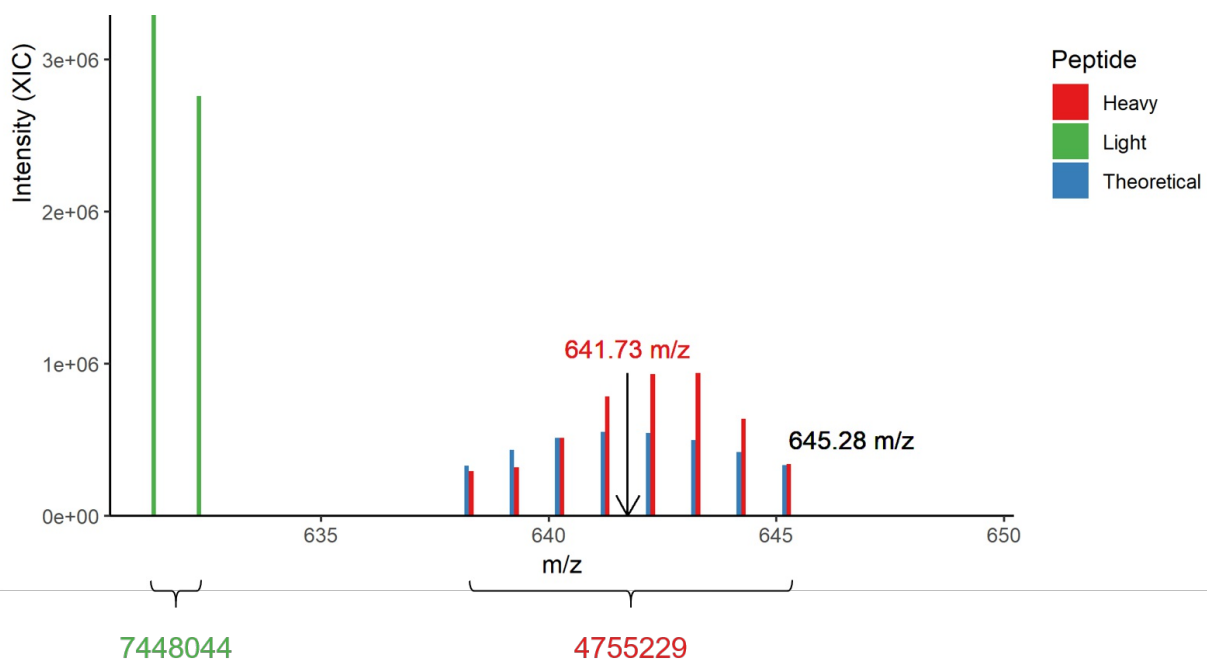

Figure 2

Bioinformatics Pipeline for Calculating RIA and Labeling Ratio. A) Work ow for extracting light and heavy peptide features from the raw MS les. B) Example of how RIA and LR were calculated. Once the light peptide feature was extracted from the raw MS le, a theoretical isotopic distribution (blue lines) was determined and compared against the observed peaks to extract the heavy features. Once a match was found, the $\mathrm{m} / \mathrm{z}$ value from the center of the theoretical distribution was chosen for RIA calculation. LR was simply the sum of the peaks after the forth $\mathrm{m} / \mathrm{z}$ peak divided by the total sum of the light and heavy features.

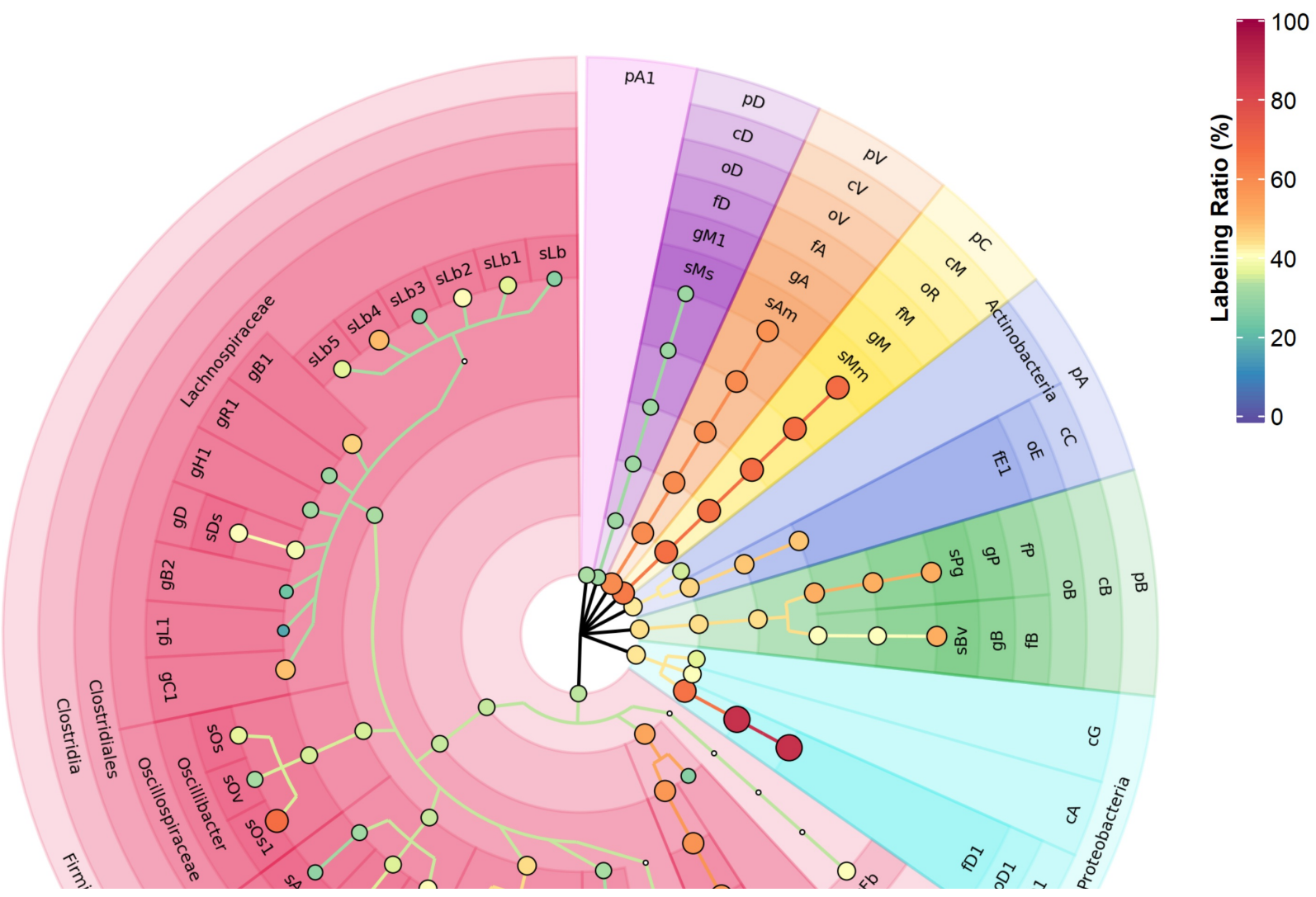




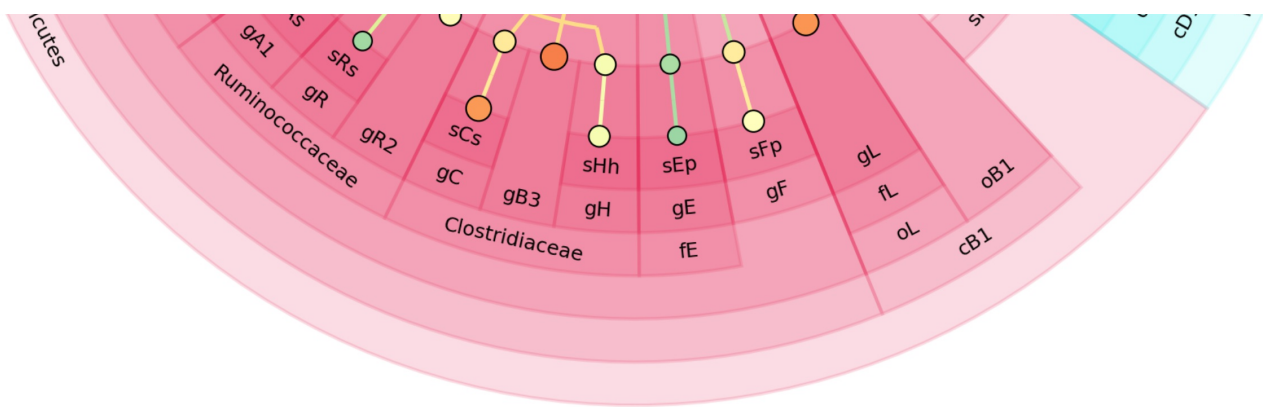

Annotation Legend:

pA: Actinobacteria

pA1: Ascomycota

pB: Bacteroidetes

pC: Chordata

pD: Deferribacteres

pV: Verrucomicrobia

cA: Alphaproteobacteria

cB: Bacteroidia

cB1: Bacilli

cC: Coriobacteriia

cD: Deferribacteres

cD1: Deltaproteobacteria

cG: Gammaproteobacteria

cM: Mammalia

cV: Verrucomicrobiae

oB: Bacteroidales

oB1: Bacillales

oD: Deferribacterales

oD1: Desulfovibrionales
oE: Eggerthellales oL: Lactobacillales oR: Rodentia

oV: Verrucomicrobiales

fA: Akkermansiaceae

fB: Bacteroidaceae

fD: Deferribacteraceae

fD1: Desulfovibrionaceae

$\mathrm{fE}$ : Eubacteriaceae

fE1: Eggerthellaceae

$\mathrm{fL}$ : Lactobacillaceae

fM: Muridae

fP: Porphyromonadaceae

gA: Akkermansia

gA1: Anaerotruncus

gB: Bacteroides

gB1: Blautia

gB2: Butyrivibrio

gB3: Butyricicoccus
gC: Clostridium gC1: Coprococcus gD: Dorea

gE: Eubacterium gF: Flavonifractor $\mathrm{gH}$ : Hungatella $\mathrm{gH} 1$ : Herbinix

gL: Lactobacillus

gL1: Lachnoclostridium

gM: Mus

gM1: Mucispirillum

gP: Parabacteroides

gR: Ruminiclostridium

gR1: Roseburia

gR2: Ruminococcus

sAm: Akkermansia muciniphila

sAs: Anaerotruncus sp. G3(2012)

sBv: Bacteroides vulgatus

sCs: Clostridium sp. ASF502
sDs: Dorea sp. 5-2

sEp: Eubacterium plexicaudatum sFb: Firmicutes bacterium ASF500 sFp: Flavonifractor plautii sHh: Hungatella hathewayi sLb: Lachnospiraceae bacterium 10-1 sLb1: Lachnospiraceae bacterium A4 sLb2: Lachnospiraceae bacterium 28-4 sLb3: Lachnospiraceae bacterium COE1 sLb4: Lachnospiraceae bacterium M18-1 sLb5: Lachnospiraceae bacterium A2 sMm: Mus musculus

sMs: Mucispirillum schaedleri sOs: Oscillibacter sp. 1-3

sOs1: Oscillibacter sp. KLE 1745

sOv: Oscillibacter valericigenes sPg: Parabacteroides goldsteinii sRs: Ruminiclostridium sp. KB18

Figure 3

Labeling Ratio of Proteins in Mouse Microbiome on Day 43. Phylogenetic tree where size and color of the nodes relates to the LR of the taxon; color of the cells corresponds to the phylum lineage; and the transparency denotes the rank. For nodes with annotations, the key follows the convention: [rst letter of phylogenetic rank][initials][unique identier]. Nodes are reported when the number of distinct peptides is greater than 3 and the adjusted $p$ value from a one-sample t-test is below 0.05 .

COG Category Legend:

Z: Cytoskeleton

$\mathrm{N}$ : Cell motility

K: Transcription

$\mathrm{V}$ : Defense mechanisms

W: Extracellular structures

I: Lipid transport and metabolism
: Signal transduction mechanisms

C: Energy production and conversion

E: Amino acid transport and metabolism

$F$ : Nucleotide transport and metabolism

G: Carbohydrate transport and metabolism

M: Cell wall/membrane/envelope biogenesis
$P$ : Inorganic ion transport and metabolism

$\mathrm{J}$ : Translation, ribosomal structure and biogenesis

O: Posttranslational modification, protein turnover, chaperones

Q: Secondary metabolites biosynthesis, transport and catabolism

U: Intracellular trafficking, secretion, and vesicular transport
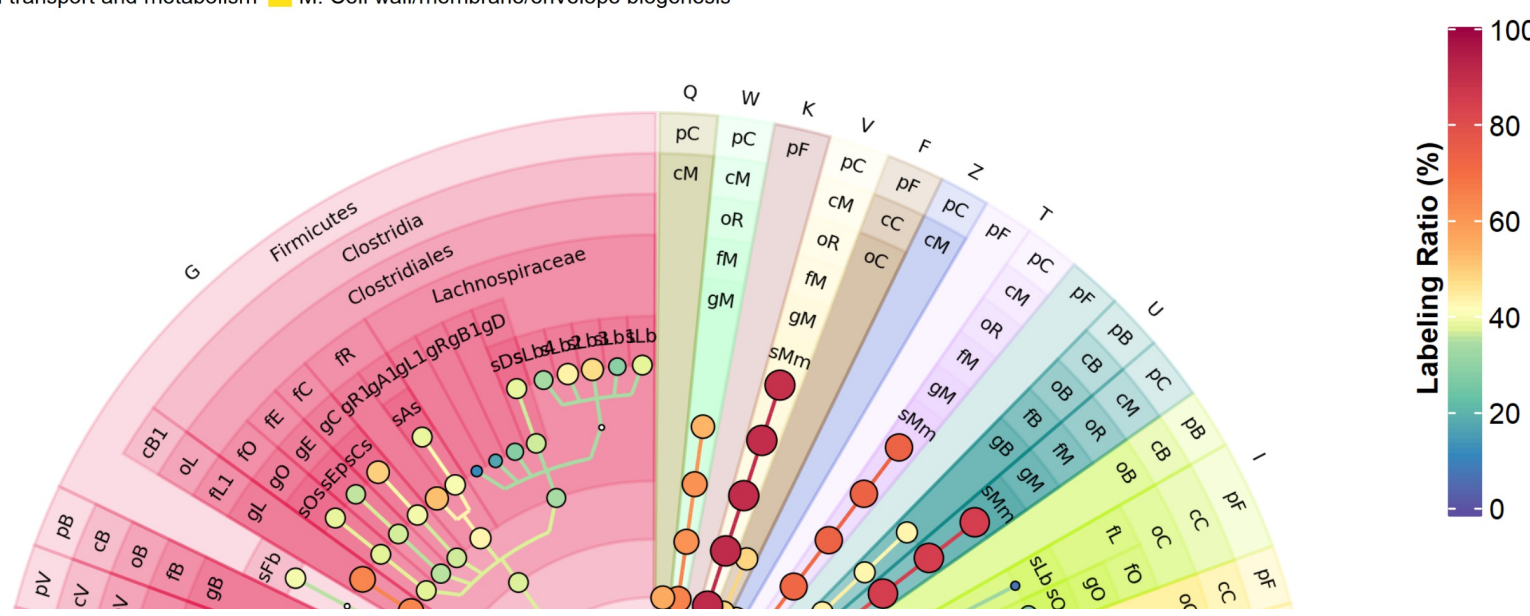


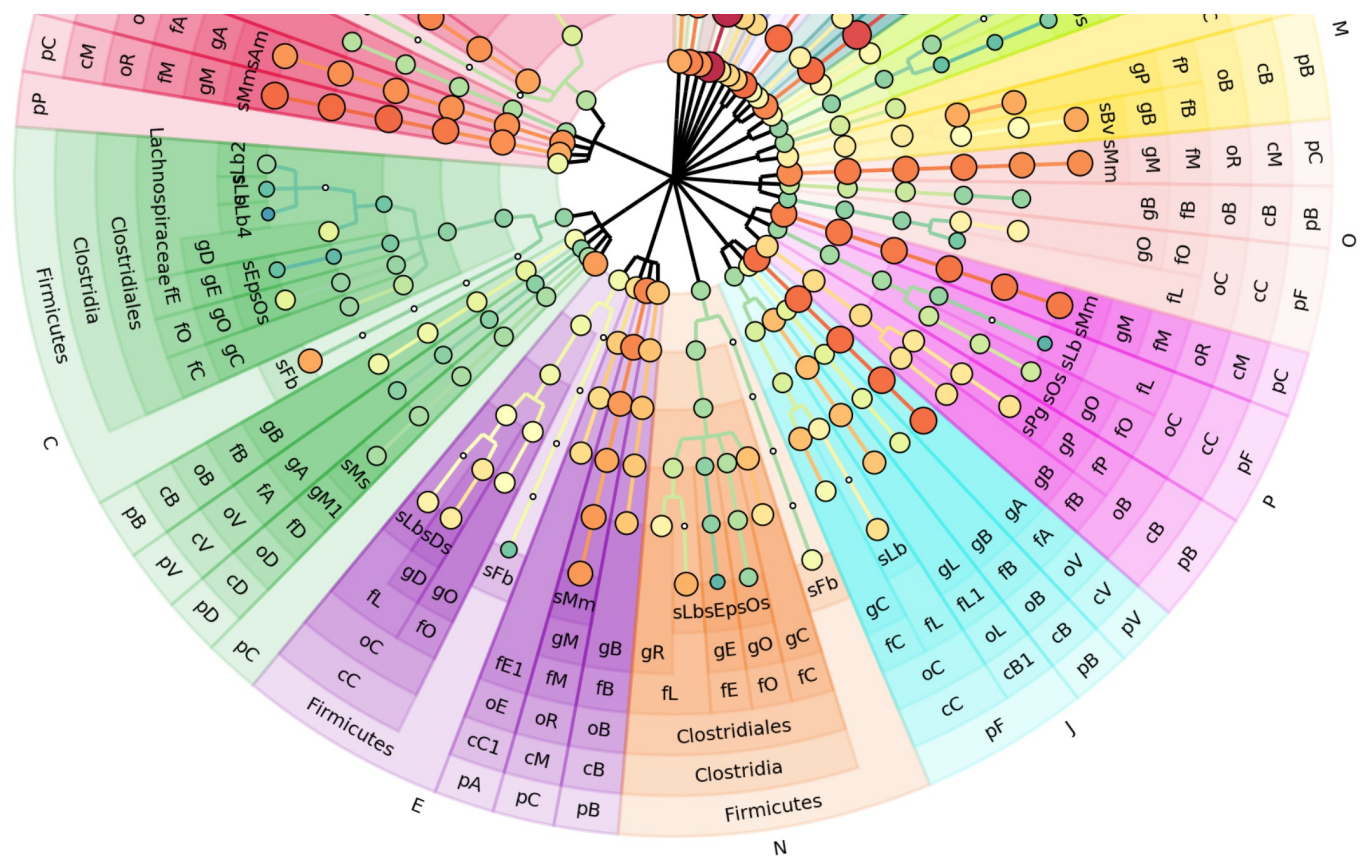

Annotation Legend:

$\mathrm{pA}$ : Actinobacteria

pB: Bacteroidetes

pC: Chordata

$\mathrm{pD}$ : Deferribacteres

pF: Firmicutes

pP: Proteobacteria

$\mathrm{pV}$ : Verrucomicrobia

$\mathrm{CB}$ : Bacteroidia

CB1: Bacilli

CC: Clostridia

CC1: Coriobacteriia

cD: Deferribacteres

cM: Mammalia

cV: Verrucomicrobiae

$\mathrm{OB}$ : Bacteroidales

oC: Clostridiales
oD: Deferribacterales

oE: Eggerthellales

oL: Lactobacillales

oR: Rodentia

oV: Verrucomicrobiales

fA: Akkermansiaceae

fB: Bacteroidaceae

fC: Clostridiaceae

fD: Deferribacteraceae

fE: Eubacteriaceae

fE1: Eggerthellaceae

fL: Lachnospiraceae

fL1: Lactobacillaceae

fM: Muridae

fO: Oscillospiraceae

fP: Porphyromonadaceae
fR: Ruminococcaceae

gA: Akkermansia

gA1: Anaerotruncus

gB: Bacteroides

gB1: Butyrivibrio

gC: Clostridium

gD: Dorea

gE: Eubacterium

gL: Lactobacillus

gL1: Lachnoclostridium

gM: Mus

gM1: Mucispirillum

gO: Oscillibacter

gP: Parabacteroides

gR: Roseburia

gR1: Ruminococcus
sAm: Akkermansia muciniphila

sAs: Anaerotruncus sp. G3(2012)

$\mathrm{sBv}$ : Bacteroides vulgatus

sCs: Clostridium sp. ASF502

sDs: Dorea sp. 5-2

sEp: Eubacterium plexicaudatum

sFb: Firmicutes bacterium ASF500

sLb: Lachnospiraceae bacterium A4

sLb1: Lachnospiraceae bacterium COE1

sLb2: Lachnospiraceae bacterium 28-4

sLb3: Lachnospiraceae bacterium A2

sLb4: Lachnospiraceae bacterium 10-1

sMm: Mus musculus

sMs: Mucispirillum schaedleri

sOs: Oscillibacter $\mathrm{sp}$. 1-3

sPg: Parabacteroides goldsteinii

Figure 4

Functional Distribution of Heavy Labeled Proteins in Mouse Gut Microbiome on Day 43. The

tree describes the functional distribution of each taxon. The size and color of the nodes

relates to the LR of the taxon; color of the cells corresponds to the functional COG category;

and the transparency denotes the rank. Nodes were annotated and reported in a similar

fashion to figure 3 . 

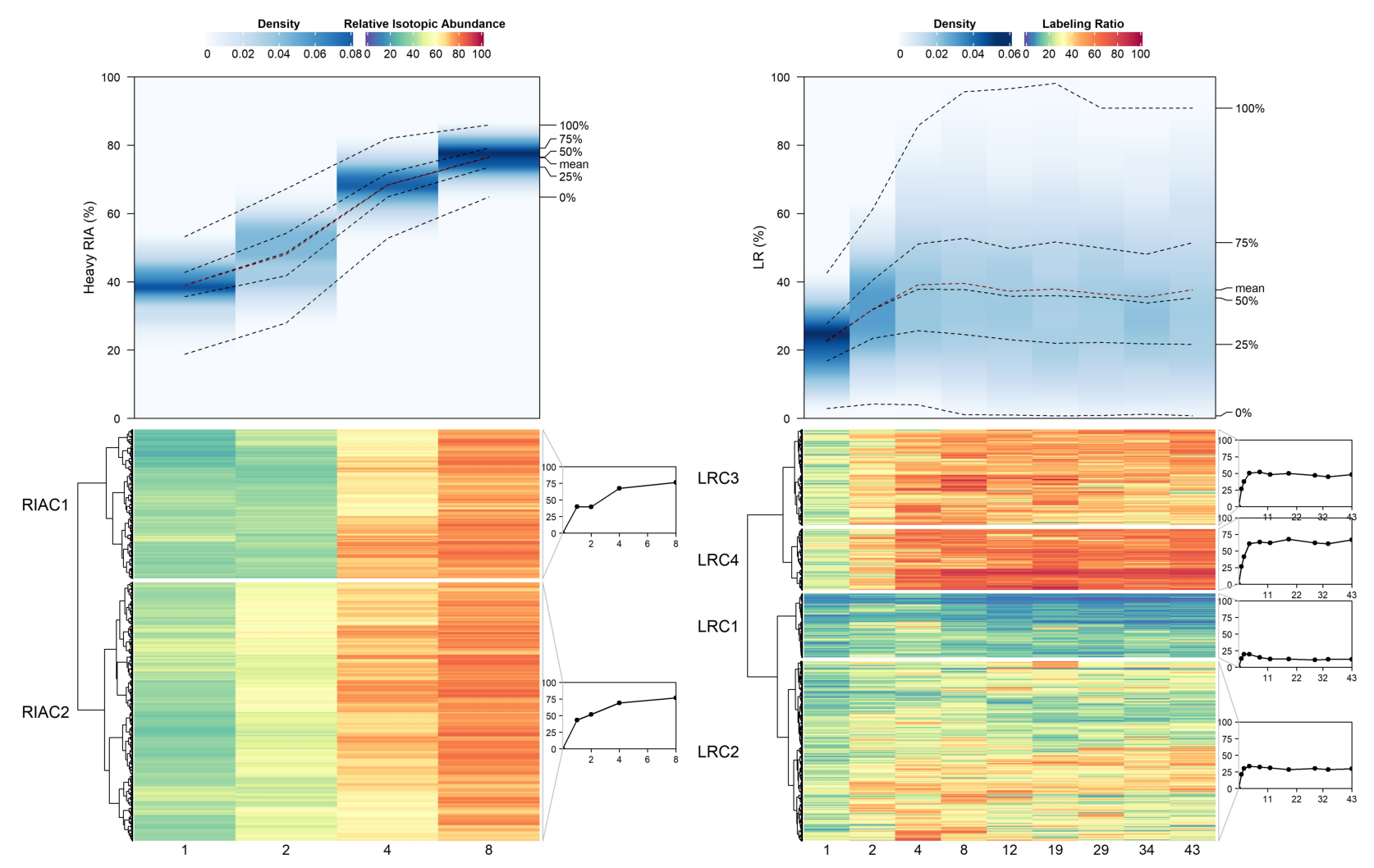

Figure 5

Isotope Incorporation Prole. A) The top graph shows the distribution of RIA at each time point. Dark blue denotes a high density and light blue denotes a low density. At the bottom is a heatmap where the rows are peptides and the columns are the days at which the RIA was recorded. B) Similar to A except that it denotes labeling ratio over time. Both heatmaps were clustered using Ward's minimum variance, as implemented in [40], and Euclidean distance. 
A
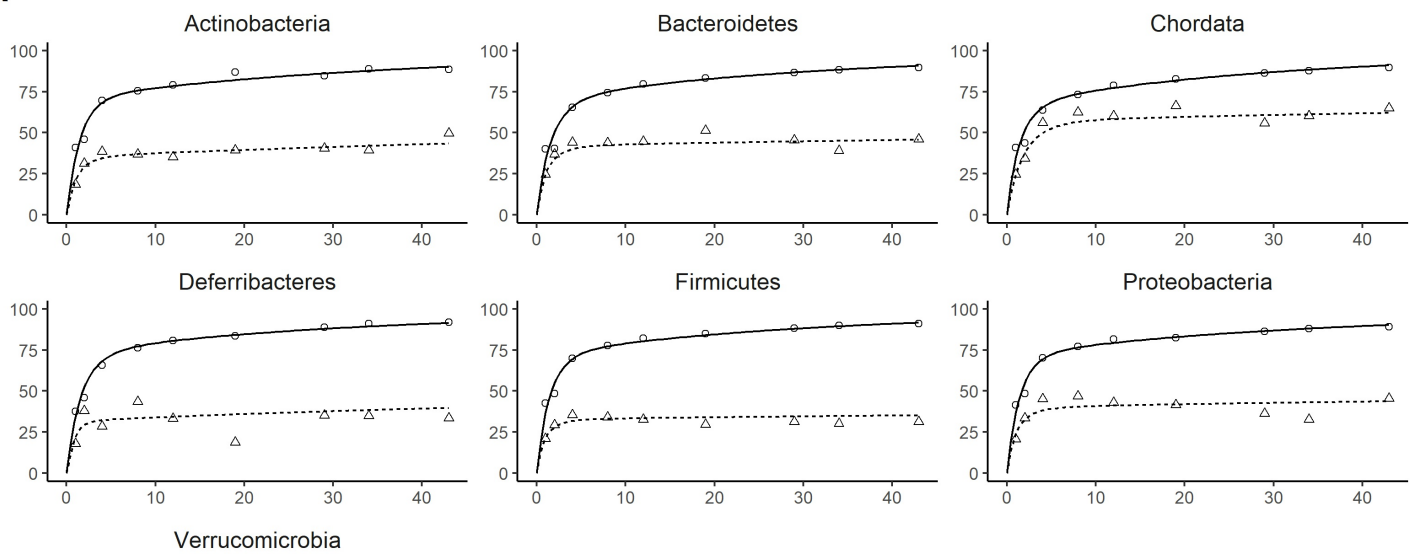

$y$-axis

$\rightarrow$ Heavy RIA (\%)

- $\triangleq$. LR (\%)

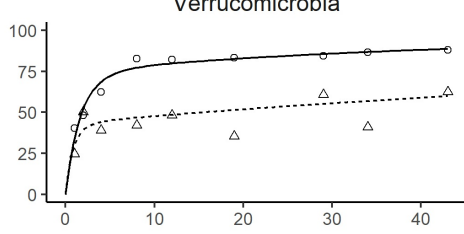

B
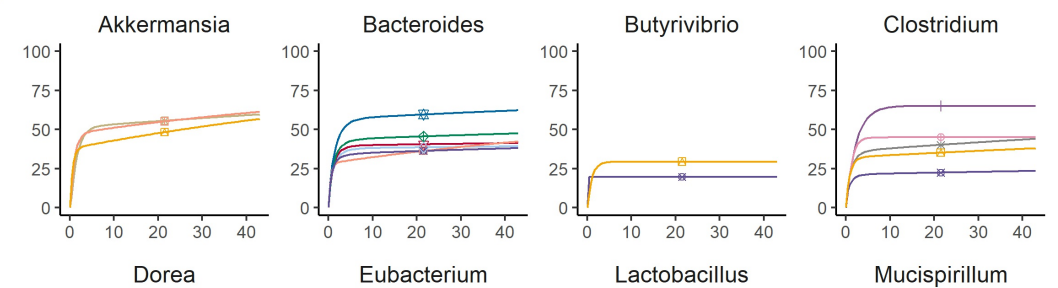

COG category

- Defense mechanisms

$\triangle$ Extracellular structures

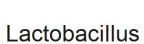

Mucispirillum

+ Nucleotide transport and metabolism

* Signal transduction mechanisms
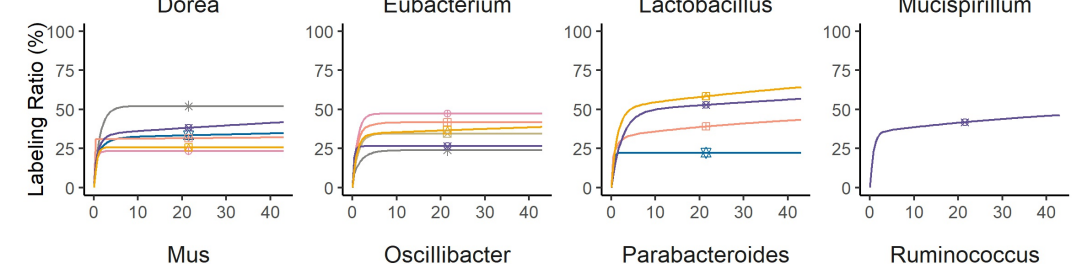

- Cell wall/membrane/envelope biogenesis

- Intracellular trafficking, secretion, and vesicular transport

- Lipid transport and metabolism

* Cell motility

$\leadsto$ Inorganic ion transport and metabolism

- Posttranslational modification, protein turnover, chaperones

\# Amino acid transport and metabolism
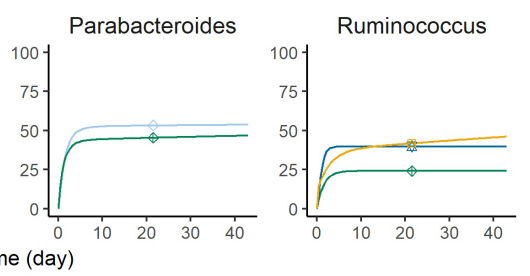

- Translation, ribosomal structure and biogenesis

* Energy production and conversion

- Carbohydrate transport and metabolism

Figure 6

Taxon-Specic RIA and LR Proles Over Time. (A) phylum level RIA and LR proles over time; (B)

Average COG LR proles over time of the 12 most abundant genera.

\section{Supplementary Files}

This is a list of supplementary files associated with this preprint. Click to download.

FigureS4.png

supplementary_information.pdf

Figures3.png

supplemental_tables.xIsx

FigureS1.png 
bmc_article.bib

ProteomeXchange_reviewer_account_details.txt

bmc_article.bbl

Figures2.png

supplementary_information.tex

Manuscript.tex

bmcart.cls

additional_file_1.pdf

vancouver.bst

bmcart-biblio.sty 